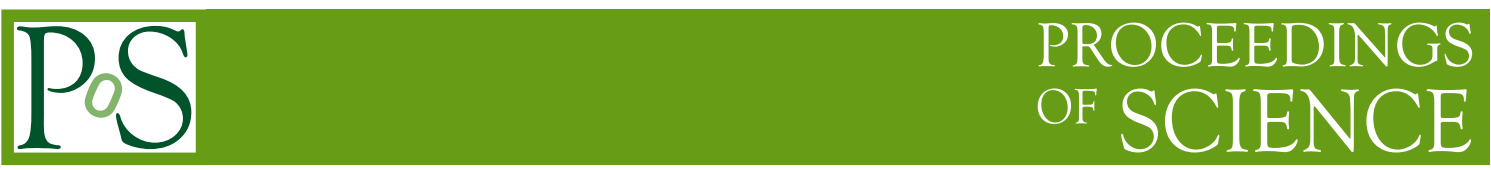

\title{
Compact radio jets and nuclear regions in active galaxies
}

\section{Andrei Lobanov}

Max-Planck-Institut für Radioastronomie, Bonn, Germany

E-mail: alobanov@mpifr-bonn.mpg.de

\begin{abstract}
VLBI observations of relativistic outflows (jets) in galactic nuclei, complemented with detailed studies made in other spectral domains, have become an effective tool for investigating the physics of nuclear regions in galaxies. High-resolution radio observations access directly the regions where the jets are formed, and trace their evolution and interaction with the nuclear environment. The emission properties, dynamics, and evolution of jets in AGN are intimately connected to the characteristics of the central supermassive black hole, accretion disk, and broad-line region (BLR) in active galaxies. Large VLBI surveys (15 GHz VLBA survey, MOJAVE) and dedicated monitoring programmes follow systematically the evolution of several hundreds of relativistic jets. These observations, combined with optical and X-ray studies, yield arguably the most detailed picture of the galactic nuclei. Recent results from studies of the nuclear regions in several active galaxies with prominent outflows are reviewed in this contribution.
\end{abstract}

The 8th European VLBI Network Symposium

September 26-29, 2006

Toruń, Poland 


\section{Introduction}

Substantial progress achieved during the past decade in studies of active galactic nuclei (see [51] for a review of recent results) has brought an increasingly wider recognition of the ubiquity of relativistic outflows (jets) in galactic nuclei [17, 84], turning them into an effective probe of nuclear regions in galaxies [46]. Emission properties, dynamics, and evolution of an extragalactic jet are intimately connected to the characteristics of the supermassive black hole, accretion disk, and broad-line region (BLR) in the nucleus of the host galaxy [51]. The jet continuum emission is dominated by non-thermal synchrotron and inverse-Compton radiation [78]. The synchrotron mechanism plays a more prominent role in the radio domain and the properties of the emitting material can be assessed using the turnover point in the synchrotron spectrum [45], synchrotron self-absorption [44], and free-free absorption in the plasma [31, 81].

High-resolution radio observations access directly the regions where the jets are formed [30] and trace their evolution and interaction with the nuclear environment 62]. Evolution of compact radio emission from several hundreds of extragalactic relativistic jets is now systematically studied with dedicated monitoring programmes and large surveys using very long baseline interferometry (VLBI) such as the $15 \mathrm{GHz} \mathrm{VLBA}^{1}$ survey [33] and MOJAVE [43]. These studies, combined with optical and X-ray studies, yield arguably the most detailed picture of the galactic nuclei [58]. Presented below is a brief summary of recent results in this field, outlining the relation between jets, supermassive black holes, accretion disks, and BLR in prominent active galactic nuclei (AGN). In this respect, this review is complementary to other recent reviews [10, 36, 58] focused on formation and propagation of extragalactic relativistic jets.

\section{Anatomy of jets}

Jets in active galaxies are formed in the immediate vicinity of the central black hole [10] and they interact with every major constituent of AGN (see Figure 1 and Table 1). The jets carry away a fraction of the angular momentum and energy stored in the accretion flow [24] or corona (in low luminosity AGN [61]) and in the rotation of the central black hole [37, 38, 72].

The production of highly-relativistic outflows requires a large fraction of the energy to be converted to Poynting flux in the very central region [73]. The efficiency of this process may depend on the spin of the central black hole [59]. The collimation of such a jet requires either a large-scale poloidal magnetic field threading the disk [75] or a slower and more massive MHD outflow launched at larger disk radii by centrifugal forces [9, 77]. A two-zone version of such a hybrid outflow model is known as the "two-fluid model" [74].

At distances of $\sim 10^{3} R_{\mathrm{g}}\left(R_{\mathrm{g}}=G M / c^{2}\right.$ is the gravitational radius of a black hole), the jets become visible in the radio regime, which makes high-resolution VLBI observations a tool of choice for probing directly the physical conditions in AGN on such small scales [30, 39]. Recent studies indicate that at distances of $10^{3}-10^{5} R_{\mathrm{g}}(\lesssim 1 \mathrm{pc})$ the jets are likely to be dominated by pure electromagnetic processes such as Poynting flux [73] or have both MHD and electrodynamic components [60]. The flowing plasma is likely to be dominated by electron-positron pairs [82,

\footnotetext{
${ }^{1}$ Very Long Baseline Array of National Radio Astronomy Observatory, USA
} 


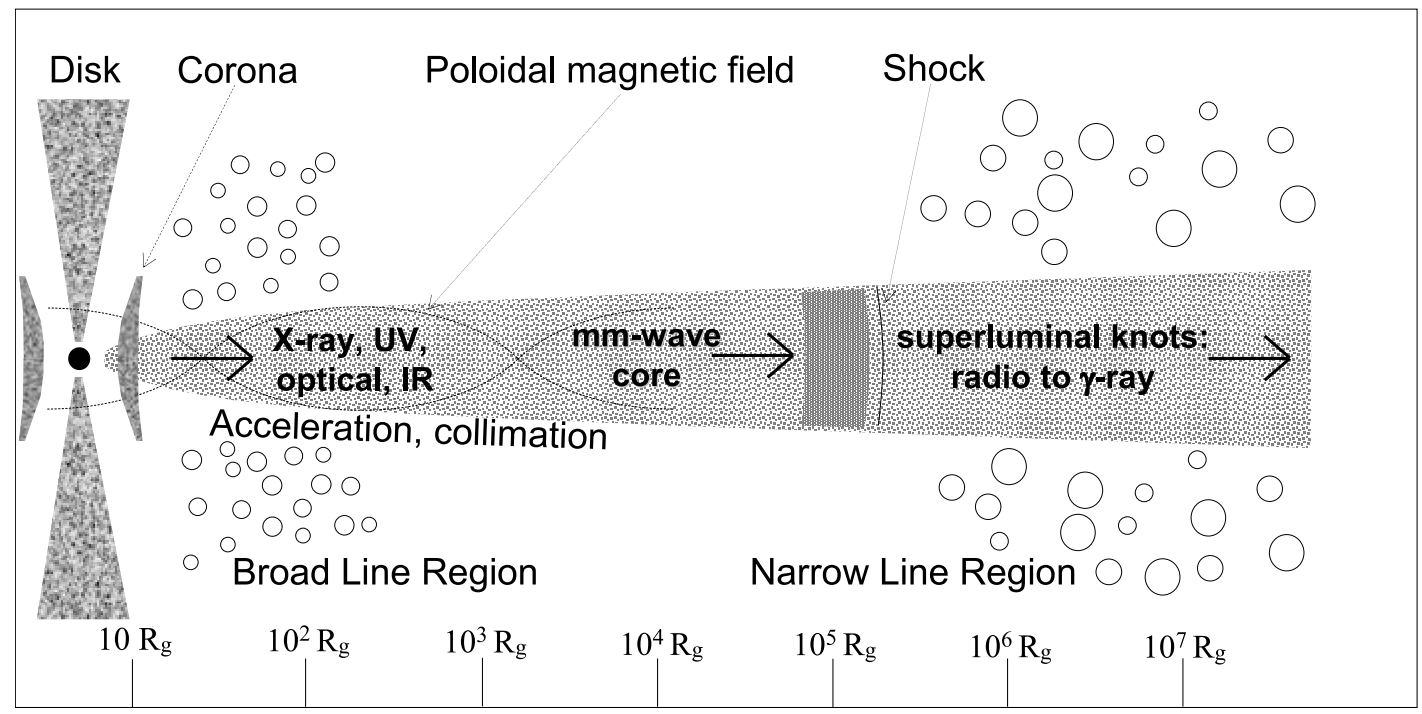

Figure 1: Basic sketch of a radio-loud AGN featuring a relativistic jet and its environment. The scale is logarithmic beyond $10 R_{\mathrm{g}}$ and vertical dimensions are not drawn to scale. Regions of the jet mainly contributing to various parts of the broadband spectrum are indicated. Adapted from [58].

Table 1: Characteristic scales in the nuclear regions in active galaxies

\begin{tabular}{rccccc}
\hline \hline & $l$ & $l_{8}$ & $\theta_{\mathrm{Gpc}}$ & $\begin{array}{c}\tau_{c} \\
{[\mathrm{yr}]}\end{array}$ & $\begin{array}{c}\tau_{\mathrm{orb}} \\
{[\mathrm{yr}]}\end{array}$ \\
\hline Event horizon: & $1-2$ & $\left.10_{\mathrm{g}}\right]$ & $5 \times 10^{-6}$ & 0.0001 & 0.001 \\
Ergosphere: & $1-2$ & $10^{-5}$ & $5 \times 10^{-6}$ & 0.0001 & 0.001 \\
Corona: & $10^{1}-10^{2}$ & $10^{-4}-10^{-3}$ & $5 \times 10^{-4}$ & $0.001-0.01$ & $0.2-0.5$ \\
Accretion disk: & $10^{1}-10^{3}$ & $10^{-4}-10^{-2}$ & 0.005 & $0.001-0.1$ & $0.2-15$ \\
Jet formation: & $>10^{2}$ & $>10^{-3}$ & $>5 \times 10^{-4}$ & $>0.01$ & $>0.5$ \\
Jet visible in the radio: & $>10^{3}$ & $>10^{-2}$ & $>0.005$ & $>0.1$ & $>15$ \\
Broad-line region: & $10^{2}-10^{5}$ & $10^{-3}-1$ & 0.05 & $0.01-10$ & $0.5-15000$ \\
Molecular torus: & $>10^{5}$ & $>1$ & $>0.5$ & $>10$ & $>15000$ \\
Narrow-line region: & $>10^{6}$ & $>10$ & $>5$ & $>100$ & $>500000$ \\
\hline
\end{tabular}

Column designation: $l$ - dimensionless scale in units of the gravitational radius, $G M / c^{2} ; l_{8}-$ corresponding linear scale, for a black hole with a mass of $5 \times 10^{8} M_{\odot} ; \theta_{\mathrm{Gpc}}-$ corresponding largest angular scale at $1 \mathrm{Gpc}$ distance; $\tau_{c}$ - rest frame light crossing time; $\tau_{\text {orb }}$ - rest frame orbital period, for a circular Keplerian orbit. Adapted from [51]

23], although a dynamically significant proton component cannot be completely ruled out at the moment [14].

The magnetic field is believed to play an important role in accelerating and collimating extragalactic jets on parsec scales [80]. How far the magnetic field dominated region extends in extragalactic jet is still a matter of debate [73]. Nevertheless, it is possible to identify three distinct regions with different physical mechanisms dominating the observed properties of the jets: 1) ultracompact jets where collimation and acceleration of the flow occurs, 2) parsec-scale flows 
dominated by relativistic shocks, and 3) large-scale jets where plasma instabilities dominate the flow.
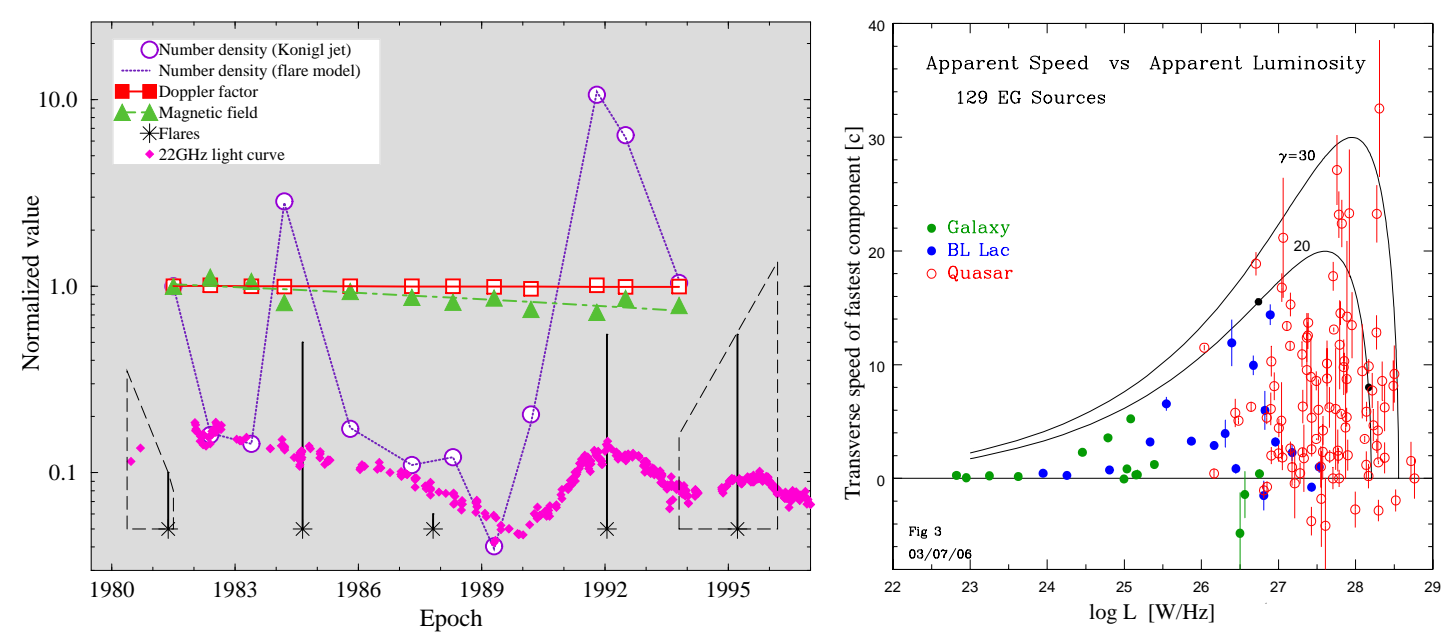

Figure 2: Left panel: Relative changes of the Doppler factor and magnetic field in the VLBI core in 3C 345, obtained by applying Königl jet model to the measured flux density and frequency of the synchrotron peak in the spectrum. All quantities are normalized to their respective values at the first epoch, $t_{0}=1981.5$. Open circles denote the particle density required for maintaining a constant Doppler factor. The dotted line shows the particle density, as represented by five exponential flares. The resulting Doppler factor (open squares) and magnetic field (filled triangles) are also shown, with lines representing linear fits to the respective quantity. The light curve of the total flux density at $22 \mathrm{GHz}$ is plotted for comparison, scaled down by a factor of 100 . Reproduced from [49]. Right panel: Values of apparent transverse speed, $\beta_{\text {app }}$, and apparent luminosity, $L$ plotted for the fastest component of 129 radio sources in the $15 \mathrm{GHz}$ VLBA survey [33]. Colours indicate different host galaxy types. The aspect curves are the loci of $\left(\beta_{\text {app }}, L\right)$ for sources with Lorentz factors $\Gamma_{\mathrm{j}}=30$ and 20 , and $L_{0}=1 \times 10^{25} \mathrm{~W} \mathrm{~Hz}^{-1}$. Reproduced from [16].

\subsection{Ultracompact jets}

Ultracompact jets observed down to sub-parsec scales typically show strongly variable but weakly polarized emission (possibly due to limited resolution of the observations). Compelling evidence exists for acceleration [5] and collimation [30, 39, 40] of the flows on these scales, which is most likely driven by the magnetic field [80]. The ultracompact outflows are probably dominated by electromagnetic processes [60, 73] and they become visible in the radio regime (identified as compact "cores" of jets ) at the point where the jet becomes optically thin for radio emission [44, 49]. The ultracompact jets do not appear to have strong shocks [45] and their basic properties are successfully described by quasi-stationary flows [35]. The evolution and variability of ultracompact jets (Figure 2, left panel) can be explained by smooth changes in particle density of the flowing plasma, associated with the nuclear flares in the central engine [49]. Intrinsic brightness temperatures of the ultracompact jets are estimated to be $(1-5) \times 10^{11} \mathrm{~K}$ [54], implying that the energy losses are dominated by the inverse-Compton process [32].

Quasi-periodic variability of the radio emission from the ultracompact jets is most likely related to instabilities and non-stationary processes in the accretion disks around central black holes 


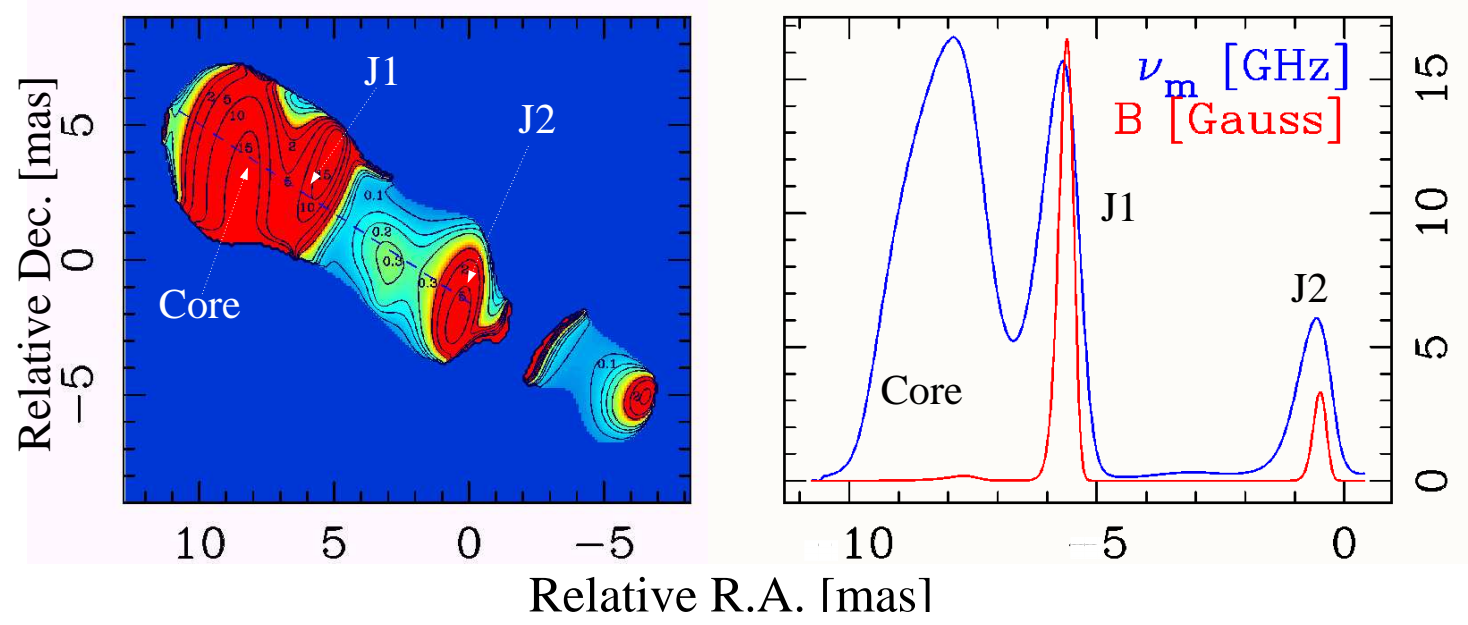

Figure 3: Left panel: Distribution of the synchrotron turnover frequency, $v_{\mathrm{m}}$, in the jet in 3C 273. The distribution is obtained from multifrequency VLBI observations and shows three regions of increased $v_{\mathrm{m}}$ : one is in the "core" of the jet and the other two are coincident with two bright superluminal features in the jet (denoted J1 and J2). Right panel: Profiles of the turnover frequency (blue) and magnetic field strength (red) taken in the jet, along the dashed line shown in the left panel. The low magnetic field in the core region indicates that the field is tangled or intrinsically weak there. The magnetic field profile shows strong spikes at the locations of the features $\mathbf{J} 1$ and $\mathbf{J} 2$, implying that these are most likely due to magnetic field compression in strong shocks propagating in the jet. Adapted from [52].

in AGN [26, 48]. Alternative explanations involve binary black hole systems in which flares are caused by passages of the secondary through the accretion disk around the primary [27, 42]. These models, however, require very tight binary systems, with orbits of the secondary lying well within $10^{3} a$ Schwarzschild radii of the primary (between 20 and 100 Schwarzschild radii, in the celebrated case of OJ 287 [42]). This poses inevitable problems for maintaining an accretion disk around the primary (for massive secondaries [51]) or rapid alignment of the secondary with the plane of the accretion disk (for small secondaries [28]). Discrepancy between the predicted and actual epoch of the latest outburst in OJ 287 [79] indicates further that the observed behaviour is not easy to be reproduced by a binary black hole scenario and it is indeed more likely to result from a quasi-periodic process in the disk, similarly to the flaring activity observed in 3C 345 [48].

\subsection{Parsec-scale flows: shocks and instabilities}

Parsec-scale outflows are characterized by pronounced curvature of trajectories of superluminal components [33, 49, rapid changes of velocity and flux density, and predominantly transverse magnetic field [29]. Statistical studies of speed and brightness temperature distributions observed in the superluminal features propagating on parsec scales indicate that the jet population has an envelope Lorentz factor of $\approx 30$ and an unbeamed luminosity of $\sim 1 \times 10^{25} \mathrm{~W} \mathrm{~Hz}^{-1}$ [16] (right panel of Figure 凤), with the brightness temperature of the emitting plasma reaching $\sim 5 \times 10^{10} \mathrm{~K}[54]$, which is close to the equipartition limit [70].

Relativistic shocks are expected to be prominent on these scales, which is manifested by strong polarization [71] and rapid evolution of the turnover frequency of synchrotron emission [52]. Map- 

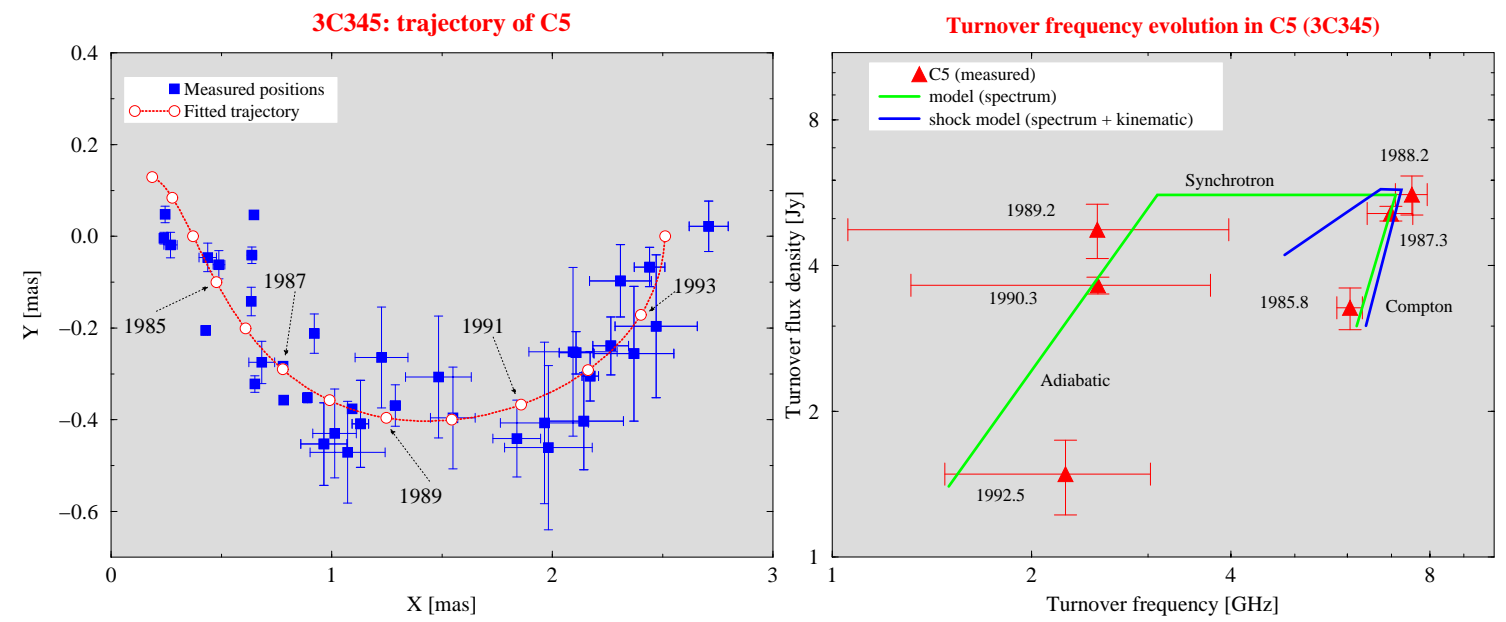

Figure 4: Left panel: The observed trajectory of $\mathrm{C} 5$ in the plane of the sky. The dotted line represents the combined polynomial fits to the component's $x$ and $y$ offsets from the core. Open circles mark the locations on the trajectory which are equally spaced in time at an interval of 1 year. Right panel: Evolution of the synchrotron turnover changes in C5. The dotted line shows an evolutionary track consistent with different stages of the evolution of the shock (Compton-loss, synchrotron-loss, and adiabatic-loss stage). The dotdashed line shows how the original fit for C5 must be changed to satisfy also the observed trajectory of the component (shown in the left panel). The shock description can only be applied at early stages of the component evolution, indicating that the shock should dissipate rapidly, on scales of $\lesssim 100 \mathrm{pc}$. Adapted from [49].

ping the turnover frequency distribution provides also a sensitive diagnostic of shocks and plasma instabilities in relativistic flows [45]. Shocks are particularly evident in the profiles of magnetic field obtained from the turnover frequency images (see Figure 3).

Complex evolution of shocked regions is revealed in observations [ [19, 29, 49] and numerical simulations [3] of parsec-scale outflows. However, the shocks are shown to dissipate rapidly [49] and shock-dominated regions are not likely to extend beyond $\sim 100 \mathrm{pc}$. This can be exemplified by the kinematic and spectral evolution observed in the jet component C5 in 3C 345 (Figure $\bigoplus$ ). The shock model can reproduce the observed spectral and kinematic changes only during the initial stages of the component evolution. This implies that strong shocks should dissipate rapidly in the jets, on scales of $\lesssim 100 \mathrm{pc}[49]$.

Observed morphologies of parsec-scale jets and trajectories of superluminal features propagating in the jets are often described in terms of a helical geometry [76], with helicity arising from some periodic process in the nucleus. Jet precession, both in single [55] and binary [13] black hole systems, have been commonly sought to be responsible for the observed helicity on parsec scales, but it may lead to rather non-physical parameters of the nuclear region (see [48] for discussion). It is more likely that the jet precession in AGN acts on time scales of $\gtrsim 10^{4}$ years and manifests itself in kiloparsec-scale structures [21] where it also becomes affected by the motion of the host galaxy [83]. Determining the precession parameters from observations of parsec-scale structures requires detailed description of relativistic effects and source geometry. This yields much longer orbital and precession periods [48] than those obtained from associating quasi-periodic changes observed on these scales directly with precession [13]. On parsec scales, the observed helical patterns 


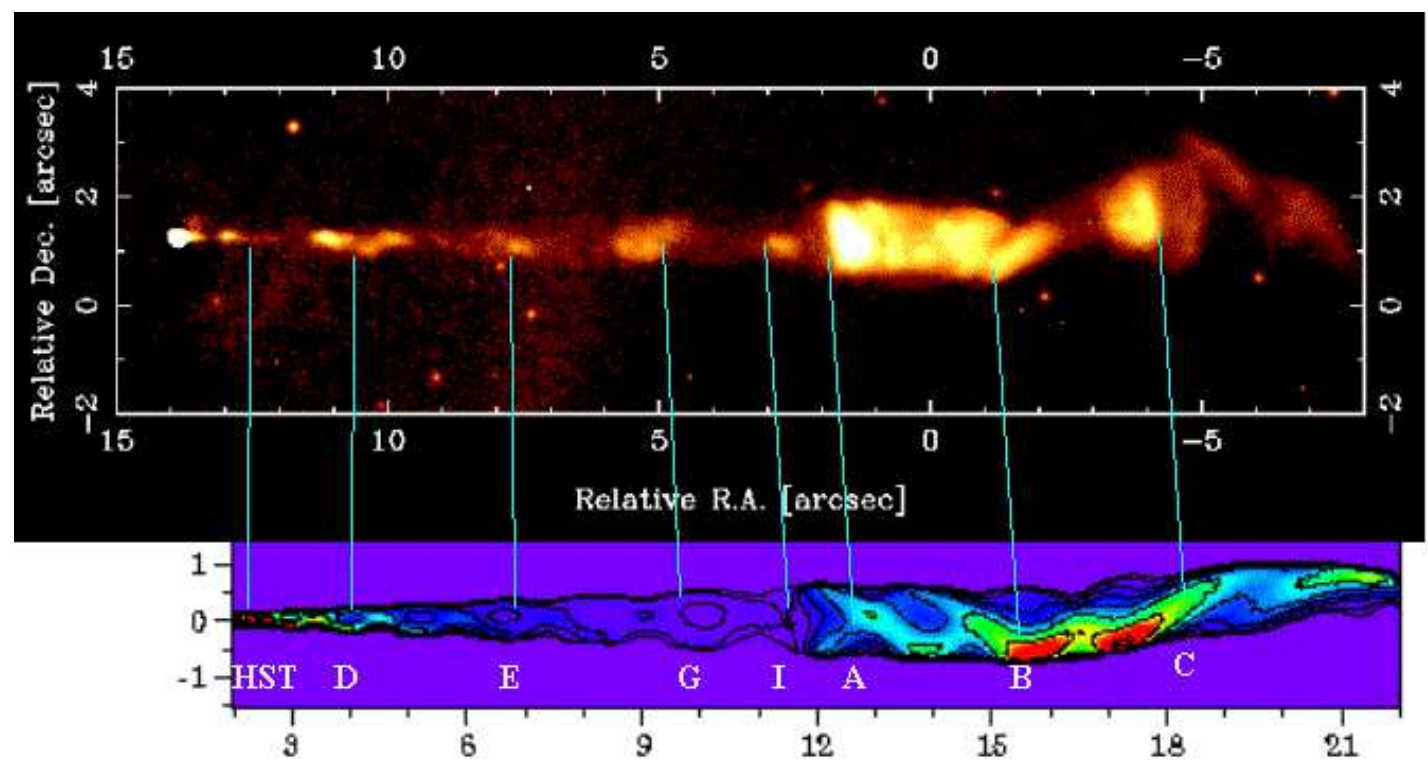

Figure 5: Top: HST image of the jet in M87. Bottom: Model based on linear description of KelvinHelmholtz instability developing in the jet. Line-of-sight synchrotron intensity image and contours at a jet viewing angle of $40^{\circ}$ including all light travel time effects and time delays. The beginning point is about $2^{\prime \prime}$ out from the nucleus with the end point at about $22^{\prime \prime}$. The spatial twist is such that the twisted flow is more towards the observer at the bottom of the jet and is more away at the top of the jet. Thus, knots A and C would be Doppler de-boosted and knot B would be Doppler boosted. Reproduced from [53].

may be produced not exclusively by precession but also by other processes, including KelvinHelmholtz instability [22] and rotation of the flow [11]. The flow rotation is particularly relevant for explaining periodic changes of the "ejection angle" (flow direction in the immediate vicinity of the VLBI core on sub-milliarcsecond scales) reported in several prominent objects [1, 2. 63.

\subsection{Large-scale jets}

On scales larger than $\sim 100 \mathrm{pc}$, instabilities (most importantly, Kelvin-Helmholtz instability) determine at large the observed structure and dynamics of extragalactic jets [50, 53, 69, The elliptical mode of the instability is responsible for appearance of thread-like features in the jet interior, while overall oscillations of the jet ridge line are caused by the helical surface mode. Successful attempts have been made to represent the observed brightness distribution of radio emission on these scales, using linear perturbation theory of Kelvin-Helmholtz instability [53] and a spinesheath (analogous to the two-fluid) description of relativistic flows [12, 41]. Figure 5 illustrates application of linear Kelvin-Helmholtz model to reproducing the radio brightness distribution in the jet of M87 [53]. Non-linear evolution of Kelvin-Helmholtz instability [66, 67] and stratification of the flow [68] play important roles in large-scale jets. Similarly to stellar jets, rotation of the flow is expected to be important for extragalactic jets [18], but observational evidence remains very limited on this issue.

\section{Jets and nuclear regions in AGN}

A number of recent studies have used jets to probe physical conditions in the central regions 

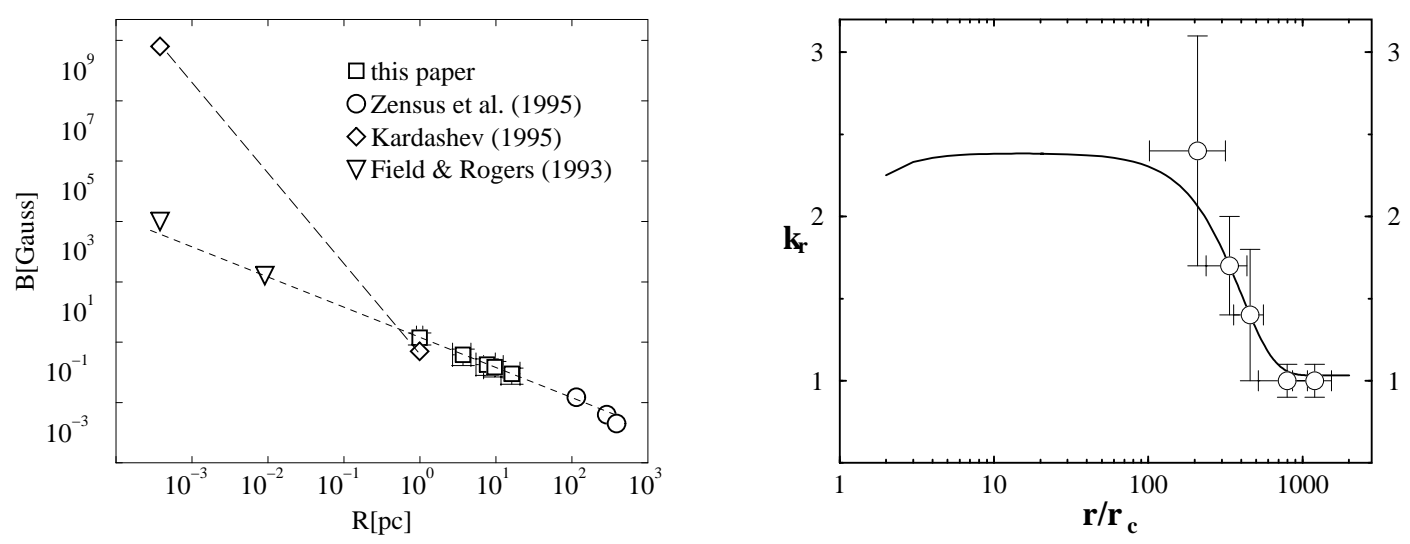

Figure 6: Left panel: Magnetic field distribution in the jet in 3C 345. Squares show the magnetic field in the compact jet derived from the frequency-dependent shift of the core. Circles are the homogeneous synchrotron model estimates of magnetic field in the extended jet components. Triangles show the characteristic magnetic field values from a model of magnetized accretion disk. Diamonds are the theoretical estimates for the dipole magnetic field around a supermassive rotating black hole. Right panel: Opacity in the jet in 3C 309.1. Circles are the measured values of the core shift power index $k_{\mathrm{r}}$ in 3C 309.1 at different frequencies; solid line shows changes of $k_{\mathrm{r}}$ due to pressure gradients in the BLR clouds supported by thermal pressure and maintaining a mass distribution with spherically symmetrical gravitational potential. The cloud region extends up to $400 r_{\mathrm{s}}\left(r_{\mathrm{s}}\right.$ refers to the distance at which the jet becomes supersonic). The equipartition regime is approached at the outer boundary of the cloud region, with $k_{\mathrm{r}}=1$. Significant deviations from the equipartition are seen on smaller scales, resulting in stronger self-absorption in the inner parts of the jet. Reproduced from [44].

of AGN. These studies have probed physical conditions in the compact relativistic flows, properties of atomic and molecular material in circumnuclear regions of AGN, and connection between relativistic outflows, accretion disks, and BLRs.

\subsection{Jets and nuclear regions of AGN}

Synchrotron self-absorption and external absorption in the ultracompact jets (VLBI "cores") can be used effectively for determining the properties of the flow itself and its environment [44]. Absolute position of the core, $r_{\mathrm{c}}$, varies with the observing frequency, $v$, so that $r_{\mathrm{c}} \propto v^{-1 / k_{\mathrm{r}}},[3]$ ] If the core is self-absorbed and in equipartition, the power index $k_{\mathrm{r}}=1$ [6]. Changes of the core position measured between three or more frequencies can be used for determining the value of $k_{\mathrm{r}}$ and estimating the strength of the magnetic field, $B_{\text {core }}$, in the nuclear region and the offset, $R_{\text {core }}$, of the observed core positions from the true base of the jet (see Figure 6; left panel). The combination of these gives an estimate for the mass of the central black hole $M_{\mathrm{bh}} \approx 7 \times$ $10^{9} M_{\odot}\left(B_{\text {core }} / \mathrm{G}\right)^{1 / 2}\left(R_{\text {core }} / \mathrm{pc}\right)^{3 / 2}$.

Core shift measurements provide estimates of the total (kinetic + magnetic field) power, the synchrotron luminosity, and the maximum brightness temperature, $T_{\mathrm{b}, \max }$ in the jets. The ratio of particle energy to magnetic field energy can also be estimated, from the derived $T_{\mathrm{b}, \max }$. This enables testing the original Königl model [35] and several of its later modifications (e.g., [25, 8]). 
The known distance from the nucleus to the jet origin will also enable constraining the self-similar jet model [56] and the particle-cascade model [ [7].

Recent studies of free-free absorption in AGN indicate the presence of dense, ionized circumnuclear material with $T_{\mathrm{e}} \approx 10^{4} \mathrm{~K}$ distributed within a fraction of a parsec of the central nucleus [44, 81]. Properties of the circumnuclear material can also be studied using the variability of the power index $k_{\mathrm{r}}$ with frequency. This variability results from pressure and density gradients or absorption in the surrounding medium, most likely associated with BLR. Changes of $k_{\mathrm{r}}$ with frequency, if measured with required precision, can be used to estimate the size, particle density, and temperature of the absorbing material surrounding the jets (see Figure 6; right panel). Estimates of the black hole mass and the BLR size obtained from the core-shift measurements can be compared with the respective estimates obtained from the reverberation mapping and applications of the $M_{\mathrm{bh}}-\sigma_{\star}$ relation.

\subsection{Atomic and molecular absorption}

Opacity and absorption in the nuclear regions of AGN have been probed effectively using the non-thermal continuum emission as a background source [46]. Absorption due to several atomic and molecular species (most notably due to $\mathrm{H}, \mathrm{CO}, \mathrm{OH}$, and $\mathrm{HCO}^{+}$) has been detected in a number of extragalactic objects. $\mathrm{OH}$ absorption has been used to probe the conditions in warm neutral gas [20, 34], and $\mathrm{CO}$ and $\mathrm{H}$ I absorption have been used to study the molecular tori [15, 65] at a linear resolution often smaller than a parsec [62]. These observations have revealed the presence of neutral gas in a molecular torus in NGC 4151 and in a rotating outflow surrounding the relativistic jet in 1946+708 [64].

\subsection{Jet-disk and jet-BLR connections}

Connection between accretion disks and relativistic outflows [24] has been explored using correlations between variability of X-ray emission produced in the inner regions of accretion disks and ejections of relativistic plasma into the flow [57]. The jets can also play a role in the generation of broad emission lines in AGN. The beamed continuum emission from relativistic jet plasma can illuminate atomic material moving in a sub-relativistic outflow from the nucleus, producing broad line emission in a conically shaped region located at a significant distance above the accretion disk 沛. Magnetically confined outflows can also contain information about the dynamic evolution of the central engine, for instance that of a binary black hole system [48]. This approach can be used for explaining, within a single framework, the observed optical variability, kinematics, and flux density changes of superluminal features embedded in radio jets.

\section{Conclusion}

Extragalactic jets are an excellent laboratory for studying physics of relativistic outflows and probing conditions in the central regions of active galaxies. Recent studies of extragalactic jets show that they are formed in the immediate vicinity of central black holes in galaxies and carry away a substantial fraction of the angular momentum and energy stored in the accretion flow and rotation of the black hole. The jets are most likely collimated and accelerated by electromagnetic fields. Relativistic shocks are present in the flows on small scales, but dissipate on scales 
of $\lesssim 100$ pc. Plasma instabilities dominate the flows on larger scales. Convincing observational evidence exists, connecting ejections of material into the flow with instabilities in the inner accretion disks. In radio-loud objects, continuum emission from the jets may also drive broad emission lines generated in sub-relativistic outflows surrounding the jets. Magnetically confined outflows may preserve information about the dynamics state of the central region, allowing detailed investigations of jet precession and binary black hole evolution to be made. This makes studies of extragalactic jets a powerful tool for addressing the general questions of physics and evolution of nuclear activity in galaxies.

\section{References}

[1] Z. Abraham, E.A. Carrara: ApJ 496, 172 (1998)

[2] Z. Abraham, G.E. Romero: A\&A 344, 61 (1999)

[3] I. Agudo, J.L. Gómez, J.M. Martí, et al.: ApJ 549, 183 (2001)

[4] T.G. Arshakian, A.P. Lobanov, V.H. Chavushyan, et al.: A\&A subm. (2006), astro-ph/0512393

[5] U. Bach, M. Kadler, T.P. Krichbaum, et al.: Multi-Frequency and Multi-Epoch VLBI Study of Cygnus A. In: Future Directions in High Resolution Astronomy: The 10th Anniversary of the VLBA, ASP Conference Proceedings, Vol. 340, ed. by J. Romney, M. Reed (ASP, San Fransisco 2005) pp. 30-34

[6] R.D. Blandford, A. Königl: ApJ 232, 34 (1979)

[7] R.D. Blandford, A. Levinson: ApJ 441, 79 (1995)

[8] S.D. Bloom, A.P. Marscher: ApJ 461, 657 (1996)

[9] S.V. Bogovalov, K. Tsinganos: MNRAS 357, 918 (2005)

[10] M. Camenzind: MemSAIt 76, 98 (2005)

[11] M. Camenzind, M. Krockenberger: A\&A 255, 59 (1992)

[12] J.R. Canvin, R.A. Laing, A.H. Bridle, W.D. Cotton: MNRAS 363, 1223 (2005)

[13] A. Caproni, Z. Abraham: ApJ 602, 625 (2004)

[14] A. Celotti, A.C. Fabian: MNRAS 264, 228 (1993)

[15] J.E. Conway: New Astron. Rev. 43, 509 (1999)

[16] M.H. Cohen, M.Lister, D. Homan, et al.: ApJ, subm. (2006)

[17] H. Falcke: Rev. Mod. Astron. 14, 15 (2001)

[18] C. Fendt: A\&A 323, 999 (1997)

[19] J.L. Gómez, A.P. Marscher, A. Alberdi, et al.: ApJ 561, 161 (2001)

[20] J.R. Goikoechea, J. Martín-Piintado, J. Chernicharo: ApJ 619, 291 (2005)

[21] A.C. Gower, P.C. Gregory, W.G. Unruh, J.B. Hutchings: ApJ 262, 478 (1982)

[22] P.E. Hardee: ApJ 597, 798 (2003)

[23] K. Hirotani: ApJ 619, 73 (2005)

[24] M. Hujeirat, M. Livio, M. Camenzind, et al.: A\&A 408, 415 (2003) 
[25] D.J. Hutter, S.L. Mufson: ApJ 301, 50 (1986)

[26] I.V. Igumenschev, M.A. Abramowicz: MNRAS 303, 309 (1999)

[27] P.B. Ivanov, I.V. Igumenschev, I.D. Novikov: ApJ 507131 (1998)

[28] P.B. Ivanov, J.C.B. Papaloizou, A.G. Polnarev: MNRAS 307, 79 (1999)

[29] S.G. Jorstad, A.P. Marscher, M.L. Lister, et al.: AJ 130, 1418 (2005)

[30] W. Junor, J.A. Biretta, M. Livio: Nature 401, 891 (1999)

[31] M. Kadler, E. Ros, A.P. Lobanov, et al.: A\&A 426, 481 (2004)

[32] K.I. Kellermann, I.I.K. Pauliny-Toth: ApJ 155, 71 (1969)

[33] K.I. Kellermann, M.L. Lister, D.C. Homan, et al.: AJ, 609, 539 (2004)

[34] H.R. Klöckner, W.A. Baan: ApSS 295, 277 (2005)

[35] A. Königl: ApJ 243, 700 (1981)

[36] A. Königl: MemSAIt 77, 598 (2006)

[37] S. Koide, K. Shibata, T. Kudoh, et al.: Science 295, 1688 (2002)

[38] S.S. Komissarov: MNRAS 359, 801 (2005)

[39] T.P. Krichbaum, D.A. Graham, A. Kraus, et al.: Towards the Event Horizon - The Vicinity of AGN at Micro-Arcsecond Resolution. In: Proceedings of the 7th Symposium of the European VLBI Network, ed. by R. Bachiller, F. Colomer, J.-F. Desmurs, P. de Vicente (Observatorio Astronomico Nacional, Madrid 2004) pp. 15-18

[40] T.P. Krichbaum: this volume, (2006)

[41] R.A. Laing, A.H. Bridle: MNRAS 348, 1459 (2004)

[42] H.J. Lehto, M.J. Valtonen: ApJ 460, 207 (1996)

[43] M.L. Lister, D.C. Homan: AJ 130, 1389 (2005)

[44] A.P. Lobanov: A\&A 390, 79 (1998)

[45] A.P. Lobanov: A\&ASS 132, 261 (1998)

[46] A.P. Lobanov: MemSAItS 7, 12 (2005)

[47] A.P. Lobanov: Nuclear activity in galaxies driven by supermassive binary black holes. In: Relativistic Astrophysics and Cosmology, ESO Astrophysical Symposia, eds. B. Aschenbach, V. Burwitz, G.

Hasinger, B. Leibundgut (Springer: Heidelberg 2006) astro-ph/0606198

[48] A.P. Lobanov, J. Roland: A\&A 431, 831 (2005)

[49] A.P. Lobanov, J.A. Zensus: ApJ 521, 509 (1999)

[50] A.P. Lobanov, J.A. Zensus: Science 284, 291 (2001)

[51] A.P. Lobanov, J.A. Zensus: Active Galactic Nuclei at the Crossroads of Astrophysics. In: Exploring the Cosmic Frontier: Astrophysical Instruments for the $21^{\text {st }}$ Century, ESO Astrophysical Symp. Series, ed. by A.P. Lobanov, J.A. Zensus, C. Cesarsky, P.J. Diamond (Springer, Heidelberg 2006) pp. $147-162$

[52] A.P. Lobanov, E. Carrara, J.A. Zensus: Vistas in Astronomy 41, 253 (1997) 
[53] A.P. Lobanov, P.E. Hardee, J.A. Eilek: New Astron. Rev. 47, 629 (2003)

[54] A.P. Lobanov, T.P. Krichbaum, D.A. Graham, et al.: A\&A 364, 391 (2000)

[55] J.-F. Lu, B.-Y. Zhou: ApJ 635, L17 (2005)

[56] A.P. Marscher: PNAS 92, 11439 (1995)

[57] A.P. Marscher, S.G. Jorstad, J.L. Gómez, et al.: Nature 417, 625 (2002)

[58] A.P. Marscher: MemSAIt 76, 13 (2005)

[59] D.L. Meier: ApJ 522, 753 (1999)

[60] D.L. Meier: New Astron. Rev. 47, 667 (2003)

[61] A. Merloni, A.C. Fabian: MNRAS 332, 165 (2002)

[62] C.G. Mundell, J.M. Wrobel, A. Pedlar, et al.: ApJ 583, 192 (2003)

[63] R.L. Mutel, G.R. Denn: ApJ 623, 79 (2005)

[64] A.B. Peck, G.B. Taylor: ApJ 554, 147 (2001)

[65] A. Pedlar: ApSS 295, 161 (2004)

[66] M. Perucho, M. Hanasz, J.M. Martí, et al.: A\&A 427, 415 (2004)

[67] M. Perucho, J.M. Martí, M. Hanasz: A\&A 427, 431 (2004)

[68] M. Perucho, J.M. Martí, M. Hanasz: A\&A 443, 863 (2005)

[69] M. Perucho, A.P. Lobanov, J.M. Martí: these proceedings (2006)

[70] A.C.S. Readhead: ApJ 426, 51 (1994)

[71] E. Ros, J.A. Zensus, A.P. Lobanov: A\&A 354, 55 (2000)

[72] V. Semenov, S. Dyadechkin, B. Punsly: Science 305, 978 (2004)

[73] M. Sikora, M.C. Begelman, G.M. Madejski, et al.: ApJ 625, 72 (2005)

[74] H. Sol, G. Pelletier, E. Asseo: MNRAS 237, 411 (1989)

[75] H.C. Spruit, T. Foglizzo, R. Stehle: MNRAS 288, 333 (1997)

[76] W. Steffen, J.A. Zensus, T.P. Krichbaum, et al.: A\&A 302, 335 (1995)

[77] K. Tsinganos, S. Bogovalov: MNRAS 337, 553 (2002)

[78] S.C. Unwin, A.E. Wehrle, A.P. Lobanov, et al.: ApJ 480, 596 (1997)

[79] M.J. Valtonen, H.J. Lehto, A. Sillanpää, et al.: ApJ 643, 9 (2006)

[80] N. Vlahakis, A. Konigl: ApJ 605, 656 (2004)

[81] R.C. Walker V. Dhawan, J.D. Romney, et al.: ApJ 530, 233 (2000)

[82] J.F.C. Wardle, D.C. Homan, R. Ojha, D.H. Roberts: Nature 395, 457 (1998)

[83] L. Zaninetti: A\&A 221, 204 (1989)

[84] J.A. Zensus: Ann. Rev. Astron. Astrophys. 35, 607 (1997) 